OPEN ACCESS

Edited by:

Rosa Maria Bermudez-Cruz, National Polytechnic Institute of Mexico (CINVESTAV), Mexico

Reviewed by: Mohamed H. Abdel-Rahman,

The Ohio State University,

United States

Giovanni Gaudino,

Retired, Bellinzona, Switzerland

David Y. Lee,

University of New Mexico,

United States

*Correspondence:

Francesco Sabbatino

fsabbatino@unisa.it

Specialty section:

This article was submitted to Molecular and Cellular Oncology,

a section of the journal

Frontiers in Oncology

Received: 29 May 2020 Accepted: 30 October 2020 Published: 27 November 2020

Citation:

Sabbatino F, Liguori L, Malapelle U,

Schiavi F, Tortora V, Conti V,

Filippelli A, Tortora G, Ferrone CR and

Pepe S (2020) Case Report: BAP1 Mutation and RAD21 Amplification as

Predictive Biomarkers to PARP

Inhibitor in Metastatic Intrahepatic

Cholangiocarcinoma.

Front. Oncol. 10:567289.

doi: 10.3389/fonc.2020.567289

\section{Case Report: BAP1 Mutation and RAD21 Amplification as Predictive Biomarkers to PARP Inhibitor in Metastatic Intrahepatic Cholangiocarcinoma}

Francesco Sabbatino ${ }^{1,2 *}$, Luigi Liguori ${ }^{3}$, Umberto Malapelle ${ }^{4}$, Francesca Schiavi ${ }^{5}$, Vincenzo Tortora ${ }^{3}$, Valeria Conti ${ }^{1,6}$, Amelia Filippelli $^{1,6}$, Giampaolo Tortora ${ }^{7}$, Cristina R. Ferrone ${ }^{8}$ and Stefano Pepe ${ }^{1,2}$

\footnotetext{
${ }^{1}$ Department of Medicine, Surgery and Dentistry "Scuola Medica Salernitana", University of Salerno, Salerno, Italy, 2 Oncology Unit, University Hospital San Giovanni di Dio e Ruggi D’Aragona, Salerno, Italy, ${ }^{3}$ Department of Clinical Medicine and Surgery, University of Naples "Federico II", Naples, Italy, ${ }^{4}$ Public Health, University of Naples "Federico II", Naples, Italy, ${ }^{5}$ Familial Cancer Clinic and Oncoendocrinology, Veneto Institute of Oncology IOV-IRCCS, Padua, Italy, ${ }^{6}$ Clinical Pharmacology and Pharmacogenetics Unit, University Hospital "San Giovanni di Dio e Ruggi D'Aragona", Salerno, Italy, 7 Oncologia Unit, Fondazione Policlinico Universitario A. Gemelli IRCCS, Università Cattolica Del Sacro Cuore, Roma, Italy, ${ }^{8}$ Department of Surgery, Massachusetts General Hospital, Harvard Medical School, Boston, MA, United States
}

Introduction: Intrahepatic cholangiocarcinoma (ICC) is a rare hepatobiliary cancer characterized by a poor prognosis and a limited response to conventional therapies. Currently chemotherapy is the only therapeutic option for patients with Stage IV ICC. Due to the poor response rate, there is an urgent need to identify novel molecular targets to develop novel effective therapies. Precision oncology tests utilizing targeted nextgeneration sequencing (NGS) platforms have rapidly entered into clinical practice. Profiling the genome and transcriptome of cancer to identify potentially targetable oncogenic pathways may guide the clinical care of the patient.

Case presentation: We present a 56-year-old male patient affected with metastatic ICC, whose cancer underwent several precision oncology tests by different NGS platforms. A novel BAP1 mutation (splice site c.581-17_585del22) and a RAD21 amplification were identified by a commercial available platform on a metastatic lesion. No germline BAP1 mutations were identified. Several lines of evidences indicate that PARP inhibitor administration might be an effective treatment in presence of BAP1 and/or RAD21 alterations since both BAP1 and RAD21 are involved in the DNA repair pathway, BAP1 interacts with BRCA1 and BRCA1-mediated DNA repair pathway alterations enhance the sensitivity to PARP inhibitor administration. In this case, after failing conventional therapies, patient was treated with PARP inhibitor olaparib. The patient had a partial response according to RECIST criteria with an overall survival of 37.2 months from the time of diagnosis of his ICC. Following 11.0 months on olaparib treatment, sustained stable disease control is ongoing. The patient is still being treated with olaparib and no significant toxicity has been reported. 
Conclusion: These findings have clinical relevance since we have shown PARP inhibitor as a potential treatment for ICC patients harboring BAP1 deletion and RAD21 amplification. We have also highlighted the utility of NGS platforms to identify targetable mutations within a cancer.

Keywords: BAP1, precision oncology, cholangio carcinoma, Poly ADP ribose polymerase (PARP) inhibitor, RAD21, olaparib

\section{INTRODUCTION}

Cholangiocarcinoma (CCA) is historically classified by location into intrahepatic, perihilar (or Klatskintumor) and distal cancers. Intrahepatic cholangiocarcinoma (ICC) is the second most common primary intrahepatic tumor, with an estimated incidence of 1.6 per 100,000/year in the United States (1). Unfortunately, ICC carries an extremely poor prognosis with an overall 5-year survival of $5-15 \%$ (1). For patients with early stage ICC, surgical resection of the cancer and removal of local lymph nodes remains the only curative option (2). However, even with a complete resection, most patients succumb to both loco-regional and distant metastases (3). Unfortunately, most patients present with advanced disease. Palliative chemotherapy is of limited efficacy (4), highlighting the urgent need for novel effective therapies.

Different cancers express different oncogenic alterations which drive tumor progression. Several lines of evidences demonstrate that some of these alterations can be effectively targeted by tailored targeted agents, improving the overall survival of treated patients (5). These results have increased the use of precision oncology tests by targeted next-generation sequencing (NGS) platforms into clinical practice, to inform clinicians in making appropriate therapeutic decisions (6). Unselected ICC patients have been often included in "basket" trials (7), most of which have unfortunately failed to demonstrate

Abbreviations: AFP, alpha fetoprotein; AJCC, American Joint Committee on Cancer; ARID1A, AT-rich interactive domain-containing protein 1A; ATM, ataxia-telangiectasia mutated; ATR, ataxia telangiectasia and Rad3-related protein; BAP1, BRCA1 associated protein 1; BRAF, v-raf murine sarcoma viral oncogene homolog B1; BRCA1, Breast cancer type 1 susceptibility protein; CCA, Cholangiocarcinoma; CDK12, Cyclin Dependent Kinase 12; CHEK1-CHEK2, Checkpoint kinase 1-2; CK7, Cytokeratin 7; CK19, Cytokeratin 19; CT, computed tomography; EGFR, Epidermal Growth Factor Receptor; EMA, European Medicines Agency; ERCC1, excision repair cross-complementation group 1; FANC, Fanconi anaemia complementation group; FDA, Food and Drug Administration; FEN1, Flap endonuclease 1; FFPE, formalin fixed paraffin embedded; FGFR2, Fibroblast Growth Factor Receptor 2; 5-FU, 5-fluorouracil; HepPar1, Hepatocyte Paraffin 1; HER2, Human epidermal growth factor receptor 2; HR, homologous recombination; ICC, Intrahepatic cholangiocarcinoma; IDH1/ IDH2, Isocitrate Dehydrogenase 1/2; IHC, immunohistochemical; KRAS, Kirsten ras oncogene homolog; LOH, loss of heterozygosity; MSS, Microsatellite Stable; NBN, Nibrin; NGS, next-generation sequencing; NRAS, neuroblastoma; RAS, viral oncogene homolog; RPA1, Replication protein A $70 \mathrm{kDa}$ DNA-binding subunit; NTRK, neurotrophic receptor tyrosine kinase 1; PARP, poly ADP ribose polymerase; PCR, polymerase chain reaction; PR, partial response; PBRM1, Polybromo 1; PCR, polymerase chain reaction; PD, progression of disease; POLB, DNA Polymerase Beta; PRKDC, Protein Kinase; DNA-Activated, Catalytic Subunit; PTA, percutaneous thermal ablation; RAD51-RAD54, radiation-repair genes 51-54; RECIST, Response evaluation criteria in solid tumors; ROS1, ROS proto-oncogene 1receptor tyrosine kinase; STR, Short Tandem Repeat. a clinical benefit (7). As a result, there is a high interest to identifying oncogenic alterations in ICC to design potentially effective strategies in biomarker-enriched populations.

NGS of ICC has already allowed identification of molecular alterations which are involved in ICC carcinogenesis such as those in KRAS, BRAF, IDH1, IDH2, EGFR, FGFR2, ROS1, ARID1A, PBRM1, BRCA1, and BAP1 (8-16). FGFR kinase inhibitors have demonstrated anti-tumor activity in ICC patients harboring activating FGFR2 gene fusions (17-19). However, no effective therapeutic strategies have currently changed the standard of care of ICC patients harboring different types of alterations.

Here, we describe the case of a chemorefractory patient with ICC harboring BAP1 mutation and RAD21 amplification. The patient was successfully treated with the PARP inhibitor olaparib.

\section{CASE PRESENTATION}

In March 2017, a 56-year-old Caucasian male was admitted to San Giovanni di Dio and Ruggi D’Aragona University Hospital for mild abdominal pain and nausea. The patient's past medical history included i) Hodgkin's lymphoma of the spleen in 1987, treated with splenectomy and radiotherapy; ii) myocardial infarction in 2006, treated with coronary angioplasty; and iii) myocardial infarction in 2012, treated with multiple coronary artery bypass grafting. He was also a former-smoker. Patient did not present with any ICC risk factors including biliary lithiasis, alcoholic liver disease, chronic hepatitis $\mathrm{B}$ or $\mathrm{C}$ infections, or primary sclerosing cholangitis. His family history was negative for any inherited-familial cancers. Abdominal ultrasound and computed tomography (CT) scan revealed a $10 \mathrm{~cm}$ intrahepatic lesion in the left lobe of the liver, as well as stable right basal lung thickening (Figure 1A). The latter was already described in a previous chest CT scan. Ultrasound guided biopsy of the liver mass demonstrated ICC (CK7+, CK19+, HepPar1-, AFP-). In April 2017, the patient underwent a left hepatectomy and sub-total gastrectomy and cholecystectomy. Histological examination demonstrated a Stage II ICC with vascular invasion [TNM staging, American Joint Committee on Cancer (AJCC) $8^{\text {th }}$ edition]. Post operatively he was seen by the multidisciplinary team. Genomic analysis of NRAS, KRAS and BRAF $V 600$ by polymerase chain reaction (PCR) sequencing, as well as immunohistochemical (IHC) staining for detection of HER2 amplification were performed on ICC tumor tissue. Both analyses did not show any type of alteration (Supplementary Table 1). Further genomic testing of EGFR was performed by sanger sequencing, but no alterations were found in exons 18, 19, 20, and 
A

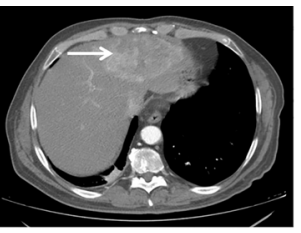

B

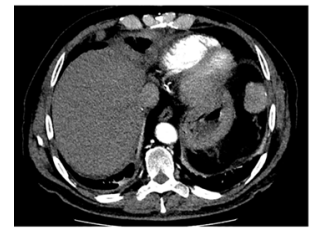

C
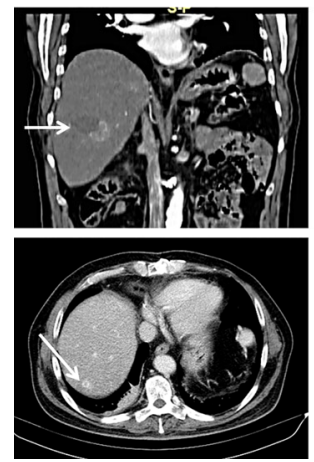
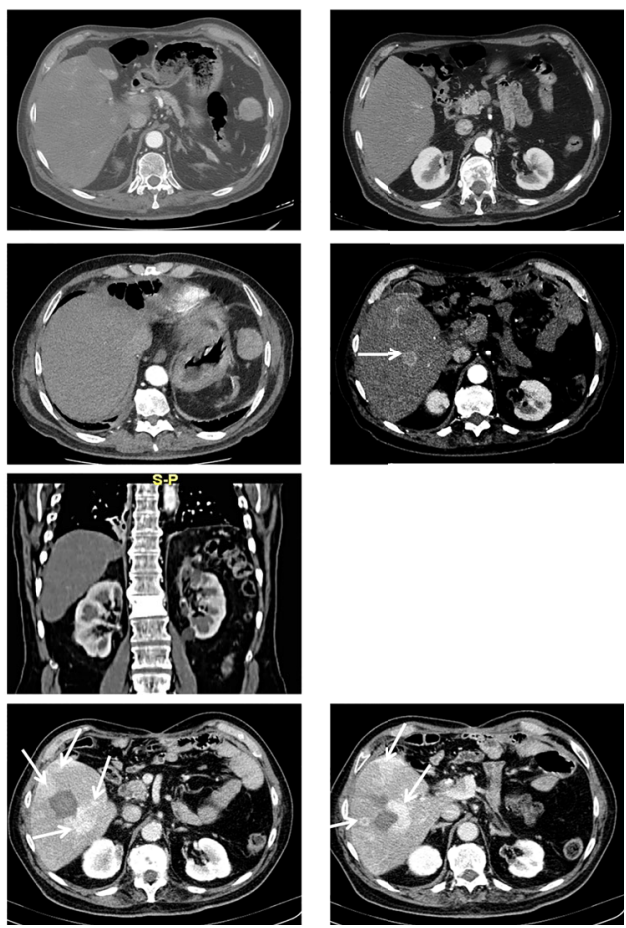

FIGURE 1 | Chest CT-scan performed at diagnosis in March 2017 (A), in October 2017 following first relapse (B), in February 2018 at tumor progression following first percutaneous thermal ablation (C), in May 2018 at tumor progression following second percutaneous thermal ablation and before starting chemotherapy with cisplatin and gemcitabine (D). Arrows indicate tumor lesion.

21 (Supplementary Table 1). In October 2017, a whole body CT scan demonstrated a $2.0 \mathrm{~cm}$ local recurrence in segment $\mathrm{V}$ of the liver (Figure 1B). Patient received a percutaneous thermal ablation (PTA) of the lesion. In February 2018, a whole body CT scan demonstrated a new $3.6 \mathrm{~cm}$ local recurrence in segment $\mathrm{V}$ of the liver, close to the previously treated lesion (Figure 1C) for which patient received a new PTA. In May 2018, a whole body CT scan demonstrated a new local recurrence in segment $\mathrm{V}$ of liver and multiple lesions in segment VII and VIII (Figure 1D). He then started a chemotherapeutic regimen with cisplatin $\left(25 \mathrm{mg} / \mathrm{m}^{2}\right)$ followed by gemcitabine $\left(1,000 \mathrm{mg} / \mathrm{m}^{2}\right)$, each administered on days 1 and 8 every 3 weeks. Due to his poor prognosis, patient requested additional testing of the ICC specimen. An IHC analysis of ROS1 rearrangements and NTRK fusions did not demonstrate any alterations (Supplementary Table 2). A Short Tandem Repeat (STR) analysis by PCR of BAT25, BAT26, D2S123, D5S346, D17S250, NR-21, and MONO-27 showed a Microsatellite Stable (MSS) tumor profile. Lastly an IHC analysis of MSH2, MSH6, PMS2, and MLH1 demonstrated no alterations of the mismatch repair system (Supplementary Table 2). Following six cycles of cisplatin and gemcitabine, in September 2018, a whole-body CT scan demonstrated a stable disease (according to RECIST criteria v 1.1). The patient received an additional PTA of the lesions in segments V, VII, and VIII of the liver. In February 2019, the CT scan demonstrated progression of disease (PD) (according to RECIST criteria $\mathrm{v} 1.1$ ) due to the development of multiple small lesions localized at the hepatic dome and around the area of previous PTA, long with a large bone metastasis to the $12^{\text {th }}$ vertebral body and a left upper lobe pulmonary nodule (Figure 2A). Based on the availability of additional formalin fixed tumor tissue obtained from a novel tumor biopsy, three different NGS platform studies were requested by the patient: Oncomine Comprehensive Assay (implemented at Istituto Tumori Milano, Milan, Italy) (Table 1), Oncofocus test [Oncologica ${ }^{\circledR}$ UK ltd (Cambridge, UK)] (Table 2) and Foundation One CDx [Foundation Medicine (Cambridge, MA)] (Table 3). Both the Oncomine Comprehensive Assay and the Oncofocus test did not detect any alterations of analyzed genes. In contrast the Foundation One CDx demonstrated the presence of a deletion in BAP1 (splice site c.581-17_585del22) and amplification of RAD21. Analysis of $B A P 1$ by sanger sequencing on primary ICC tumor tissue confirmed the presence of BAP1 (splice site 581-17_585del22) alteration (Figure 3). In contrast no alterations were identified in $B A P 1$ from nucleic acids extracted from buffy coat (Figure 3). Because of the involvement of RAD21 in the DNA repair pathway, the interaction of BAP1 with BRCA1 and the enhanced sensitivity to PARP inhibitor administration in presence of alterations in the BRCA1mediated DNA repair pathway, it was decided first to treat the patient with FOLFIRI every 2 weeks [irinotecan $180 \mathrm{mg} / \mathrm{m}^{2}$, folinic acid $400 \mathrm{mg} / \mathrm{m}^{2}$, 5-fluorouracil (5-FU) $400 \mathrm{mg} / \mathrm{m}^{2}$ intravenous infusion bolus, then 5 -FU $2400 \mathrm{mg} / \mathrm{m}^{2}$ intravenous infusion over $46 \mathrm{~h}$ ] and then to start a PARP inhibitor. FOLFIRI is a conventional second-line chemotherapy regimen for ICC. In addition, irinotecan is a DNA-damaging agent. Following six cycles of FOLFIRI, in June 2019, a whole-body CT scan demonstrated PD (Figure 2B). A third- 
A

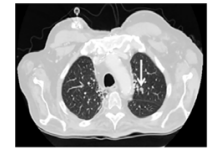

B

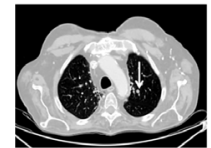

C

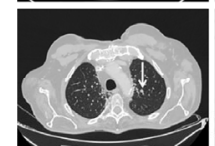

D
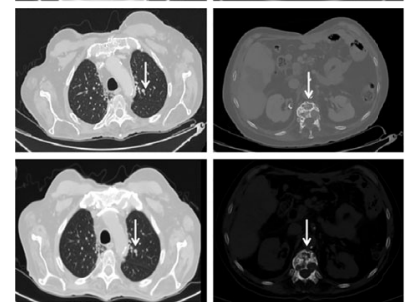
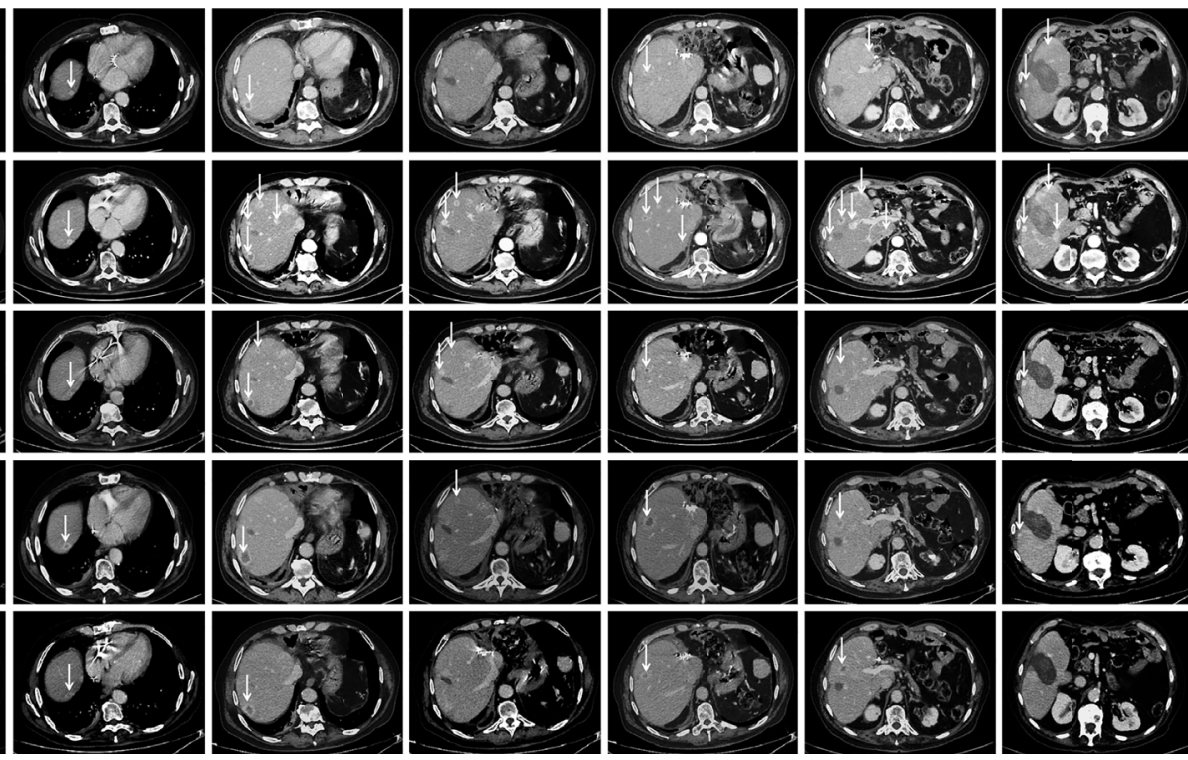
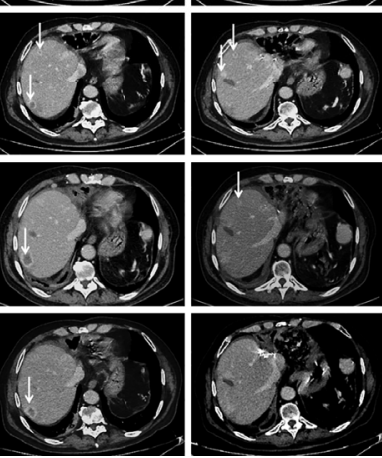
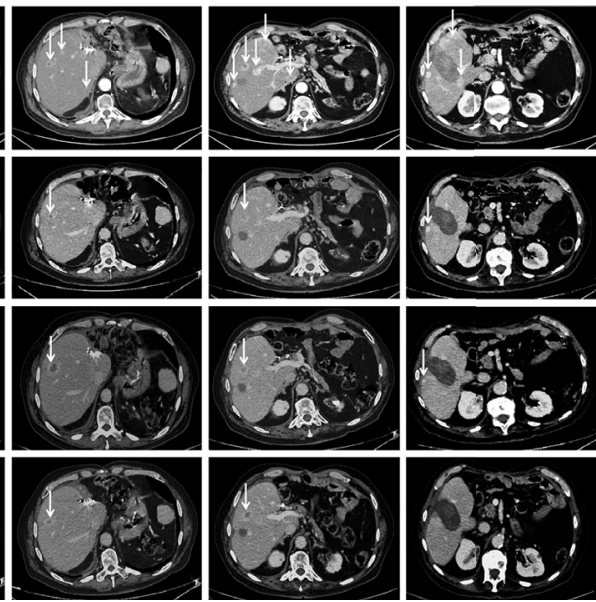

FIGURE 2 | Chest CT-scan performed at diagnosis in February 2019 at tumor progression following chemotherapy with cisplatin and gemcitabine and a third percutaneous thermal ablation and before to start treatment with FOLFIRI (A), in June 2019 at tumor progression following six cycles of FOLFIRI administration and before to start treatment with olaparib (B), in September 2019 following three cycles of olaparib (C), in November 2019 following six cycles of olaparib (D), and in February 2020 following 11 cycles of olaparib (E). Arrows indicate tumor lesion.

TABLE 1 | Oncomine Comprehensive Assay.

March $1^{\text {st }}, 2019$

NGS: Hot spot Cancer Panel with PGM (Personal Genome Machine) Ion Torrent technology [Thermo Fisher Scientific Life Technologies (Waltham, MA)]

\begin{tabular}{|c|c|c|c|c|c|c|c|c|}
\hline $\mathrm{ABL} 1$ & AKT1 & ALK & APC & ATM & BRAF & $\mathrm{CDH} 1$ & CDKN2A & CSF1R \\
\hline CTNNB1 & EGFR & ERBB2 & ERBB4 & $\mathrm{EZH} 2$ & FBXW7 & FGFR1 & FGFR2 & FGFR3 \\
\hline FLT3 & GNA11 & GNAQ & GNAS & HNF1A & HRAS & $\mathrm{IDH} 1$ & $\mathrm{IDH} 2$ & JAK2 \\
\hline JAK3 & KDR (VEGFR2) & $\mathrm{KIT}$ & KRAS & MET & MLH1 & MPL & NOTCH1 & NPM1 \\
\hline NRAS & PDGFRA & PIK3CA & PTEN & PTPN11 & $\mathrm{RB} 1$ & RET & SMAD4 & SMARCB1 \\
\hline $\mathrm{SMO}$ & SRC & STK11 & TP53 & VHL & & & & \\
\hline
\end{tabular}

Results: No hot spot mutations detected.

line therapy of off-label use with the PARP inhibitor olaparib at 800 $\mathrm{mg} /$ die and palliative radiotherapy (10 Gy) on the vertebral lesion was begun. In September 2019, a whole-body CT scan demonstrated a partial response (PR) (Figure 2C). The latter was confirmed on successive restaging scans in November 2019 (Figure 2D) and February 2020 (Figure 2E). Following 11 cycles of olaparib, the progression free survival has been 11.0 months. Currently, the patient has an overall survival of 37.2 months from the time of diagnosis of his ICC and has continued treatment with olaparib. $\mathrm{He}$ is in good health conditions and no treatment-related adverse events have been reported.

\section{DISCUSSION AND CONCLUSIONS}

Novel effective therapies are urgently needed for metastatic ICC patients. The current clinical case has provided for the first-time evidence that ICC patients carrying a BAP1 deletion and RAD21 amplification might benefit from a PARP inhibitor treatment. BAP1 is a tumor suppressor gene which modulates several pathways including cell death, cell differentiation, DNA damage response and gluconeogenesis (20-28). In mediating DNA damage response, BAP1 interacts with BRCA1 $(20,21)$. BRCA1 plays a key role in the DNA repair mechanism as well as in cell cycle regulation (29). Germline heterozygous mutations in BAP1 cause an autosomal dominant condition known as BAP1-cancer syndrome which confers a high susceptibility to the development of several malignancies including mesothelioma, uveal melanoma, renal, cholangio and breast carcinomas (30-38). In the clinical case we have described, we identified a novel mutation in BAP1 (c.581-17_585del22). The variant was somatic and not detected in the germline. We have examined several databases (Cosmic, GenBank, ClinVar) and c.581-17_585del22 mutation was not identified. Some literature data reported a similar deletion of $B A P 1$ with a pathogenic value $(39,40)$. Somatic mutations in $B A P 1$ are reported to drive 
NGS: Oncofocus test (Oncologica ${ }^{\circledR}$ UK Itd (Cambridge, UK)

\begin{tabular}{|c|c|c|c|c|c|c|c|c|c|c|c|c|c|c|}
\hline A2M & ABCB5 & ACACA & ACADM & ACBD5 & ACTG2 & ADAM32 & ADAMTS16 & AES & AFAP1 & AFF3 & AGAP3 & AGBL4 & AGGF1 & AGK \\
\hline AGTRAP & AHCYL1 & AKAP12 & AKAP13 & AKAPG & AKT1 & AKT2 & AKT3 & ALK & AP3B1 & $A R$ & ARAF & ARHGEF2 & ARID1A & ARMC10 \\
\hline ARMT1 & ASIC2 & ATAD2 & ATAD5 & ATF7IP & ATG7 & ATIC & ATM & ATP1B1 & ATR & ATRNL1 & ATRX & $A X L$ & B4GALT1 & BAG4 \\
\hline BAIAP2L1 & BAP1 & BBS9 & BCAM & BCAN & BCL2L11 & $\mathrm{BCR}$ & BEND5 & $\mathrm{BICC} 1$ & BICD2 & BIN2 & BIRC6 & BRAF & BRCA1 & BRCA2 \\
\hline BRD3 & BRD4 & BTAF1 & BTBD1 & BTF3L4 & BTK & C11orf95 & C7orf73 & C8ORF34 & C9orf153 & CAD & CAND1 & CAPRIN1 & CAPZA2 & CARS \\
\hline CASP7 & $\mathrm{CBL}$ & CCAR2 & CCDC170 & CCDC6 & CCDC88A & CCDC91 & CCND1 & CCND2 & CCND3 & CCNE1 & CCNY & CD44 & CD74 & $\mathrm{CDC} 27$ \\
\hline CDK12 & CDK2 & CDK4 & CDK5RAP2 & CDK6 & CDKN1B & CDKN2A & CDKN2B & CEL & CEP85L & CEP89 & CHD9 & CHEK1 & CHEK2 & CHTOP \\
\hline $\mathrm{ClC}$ & CIITA & CIT & CLCN6 & CLIP1 & CLIP2 & CLIP4 & CLTC & CNTLN & CNTRL & COL14A1 & COX5A & CPSF6 & CREB3L2 & CREB5 \\
\hline CREBBP & CSF1R & CTNNB1 & CUL1 & CUX1 & DAB2 & DAB2IP & DCTN1 & DDR2 & DIP2C & DNAJB1 & DTD1 & DYM & DYNC112 & DYNC2H1 \\
\hline EBF1 & EGFR & EIF3E & ELAVL3 & EML4 & EPHB2 & EPS15 & ERBB2 & ERBB3 & ERBB4 & ERC1 & ERCC2 & $E R G$ & ERLIN2 & ERP44 \\
\hline ERVK3_1 & ESR1 & ESRP1 & ETV1 & ETV4 & ETV5 & ETV6 & EZH2 & EZR & FAM114A2 & FAM131B & FAM76A & FANCA & FANCD2 & FANCl \\
\hline FA1 & FBXO28 & FBXW7 & FCHSD1 & FGF3 & FGFR1 & FGFR19 & FGFR1OP & FGFR1OP2 & FGFR2 & FGFR3 & FGFR4 & FGR & FP1L1 & FKBP15 \\
\hline FLT3 & FN1 & FNDC3B & FOXL2 & FOXP1 & FXR1 & FYCO1 & GABBR2 & GATA2 & GATM & GFPT1 & GHR & GIT2 & GLIS3 & GNA11 \\
\hline GNAl1 & GNAQ & GNAS & GNS & GOLGA4 & GOLGA5 & GOLGB1 & GOPC & GRB7 & GRHL2 & GTF2I & GTF2IRD1 & GTF3C2 & H3F3A & HACL1 \\
\hline HERPUD1 & HIP1 & HIST1H3B & HLA_A & HMGA2 & NHNF1A & HOMER1 & HOOK3 & HRAS & $\mathrm{IDH} 1$ & IDH2 & IGF1R & IRF2BP2 & JAK1 & JAK2 \\
\hline JAK3 & JAKMIP1 & KANK1 & KANK2 & KCNQ5 & KCTD1 & KCTD7 & KDELR2 & KDM7A & $\mathrm{KDR}$ & KIAA1468 & KIAA1549 & KIAA1598 & KIF5B & $\mathrm{KIT}$ \\
\hline KLC1 & KLHL7 7 & KNSTRN & KRAS & KTN1 & LMNA & LRIG3 & LRRFIP1 & LSM12 & LSM14A & LYN & MACF1 & MAD1L1 & MAGOH & MAP2K1 \\
\hline MAP2K2 & MAP2K4 & MAPK1 & MAX & MBIP & MCFD2 & MDM2 & MDM4 & MED12 & MEMO1 & MET & MGEA5 & MIR143HG & MKRN1 & MLH1 \\
\hline MPRIP & MRE11A & MRPL24 & MRPS33 & MSH2 & MSH6 & MSN & MTFHD1L & MTMR12 & MTOR & MYB & MYBL1 & MYC & MYCL & MYCN \\
\hline MYD88 & MYH13 & MYH9 & MY018A & MY05A & MYRIP & MZT1 & NACC2 & NAV1 & NBN & NCOA1 & NCOA4 & NCOR2 & NDE1 & NF1 \\
\hline NF2 & NFASC & NFIB & NFKB2 & NIN & NOL4 & NOTCH1 & NOTCH2 & NOTCH3 & $\mathrm{NOTCH} 4$ & NPC2 & NPM1 & NRAS & NRG1 & NSD1 \\
\hline NTM & NTRK1 & NTRK2 & NTRK3 & NUB1 & NUDCD3 & NUP214 & NUTM1 & OFD1 & OPHN1 & OXR1 & PALB2 & PAPD7 & PAPSS1 & PARK2 \\
\hline PAX5 & PAX8 & PCDHGA1 & PCM1 & PCNX & PDE10A & PDE4DIP & PDE7A & PDGFRA & PDGFRB & PDHX & PDP1 & PDZRN3 & PHEB & PIK3CA \\
\hline PIK3CB & PIK3R1 & PLAG1 & PLIN3 & PMS2 & POLE & POLH & PPARG & PPFIBP1 & PPHLN1 & PPL & PPM1G & PPP2R1A & PPP4R3B & PRKACA \\
\hline PRKACB & PRKAR1A & PRKG2 & PSMD11 & $\mathrm{PSPH}$ & PTCH1 & PTEN & PTPN11 & PTPN3 & PTPRK & PTPRZ1 & PWWP2A & QKI & RABEP1 & RABGAP1L \\
\hline RAC1 & RAD18 & RAD50 & RAD51 & RAD51B & RAD51C & RAD51D & RAF1 & RANBP2 & $\mathrm{RB} 1$ & RBMS3 & RBPMS & RELA & RET & $\mathrm{RHOA}$ \\
\hline RICTOR & RNF11 & RNF130 & RNF213 & RNF43 & ROS1 & RP2 & RSPO2 & RSPO3 & RUFY2- & SART3 & SCAF11 & SDC4 & SDCCAG3 & SEC16A \\
\hline SEC31A & SEC61G & SETD2 & SF3B1 & SHROOM4 & SHTN1 & SLC12A7 & SLC26A4 & SLC34A2 & SLC3A2 & SLC45A3 & SLMAP & SLX4 & SMAD4 & SMARCA4 \\
\hline SMARCB1 & SMOP & SND1 & SNHG7 & SNX19 & sox6 & SPAG9 & SPECC1 & SPECC1L & SPOP & SPTBN1 & SQSTM1 & $\mathrm{SRC}$ & SRGAP3 & SSBP2 \\
\hline STAT3 & STK11 & STK32B & STRN & STRN3 & SUGCT & TACC1 & TACC3 & TANK & TAX1BP1 & TBL1XR1 & TENM4 & TERF2 & TERT & TPM1 \\
\hline TFG & TMEM106B & TMEM178B & TMPRSS2 & TNIP1 & TNKS2 & TOP1 & TP53 & TP53BP1 & TPM3 & TPM4 & TPR & TRAF1 & TRAK1 & TRIM24 \\
\hline TRIM27 & TRIM33 & TRIM4 & TRIO & TRIP11 & TRMT61B & TSC1 & TSC2 & TSEN2 & TTLL7 & TXLNA & TYK2 & U2AF1 & UBE2L3 & UBN2 \\
\hline USP10 & VAMP2 & VCL & VOPP1 & WASF2 & WDR48 & WHSC1L1 & WIPF2 & XPO1 & YAP1 & YTHDF3 & YWHAE & ZC3HAV1 & ZCCHC8 & ZEB2 \\
\hline ZKSCAN & ZKSCAN5 & ZMYM2 & ZMYND8 & ZNF226 & ZNF703 & 30 & & & & & & & & \\
\hline
\end{tabular}

Mutations: No actionable variant detected

- Copy Number Variations: No actionable variant detected

- Fusion Genes: No actionable variant detected 
TABLE 3 | Foundation One CDX.

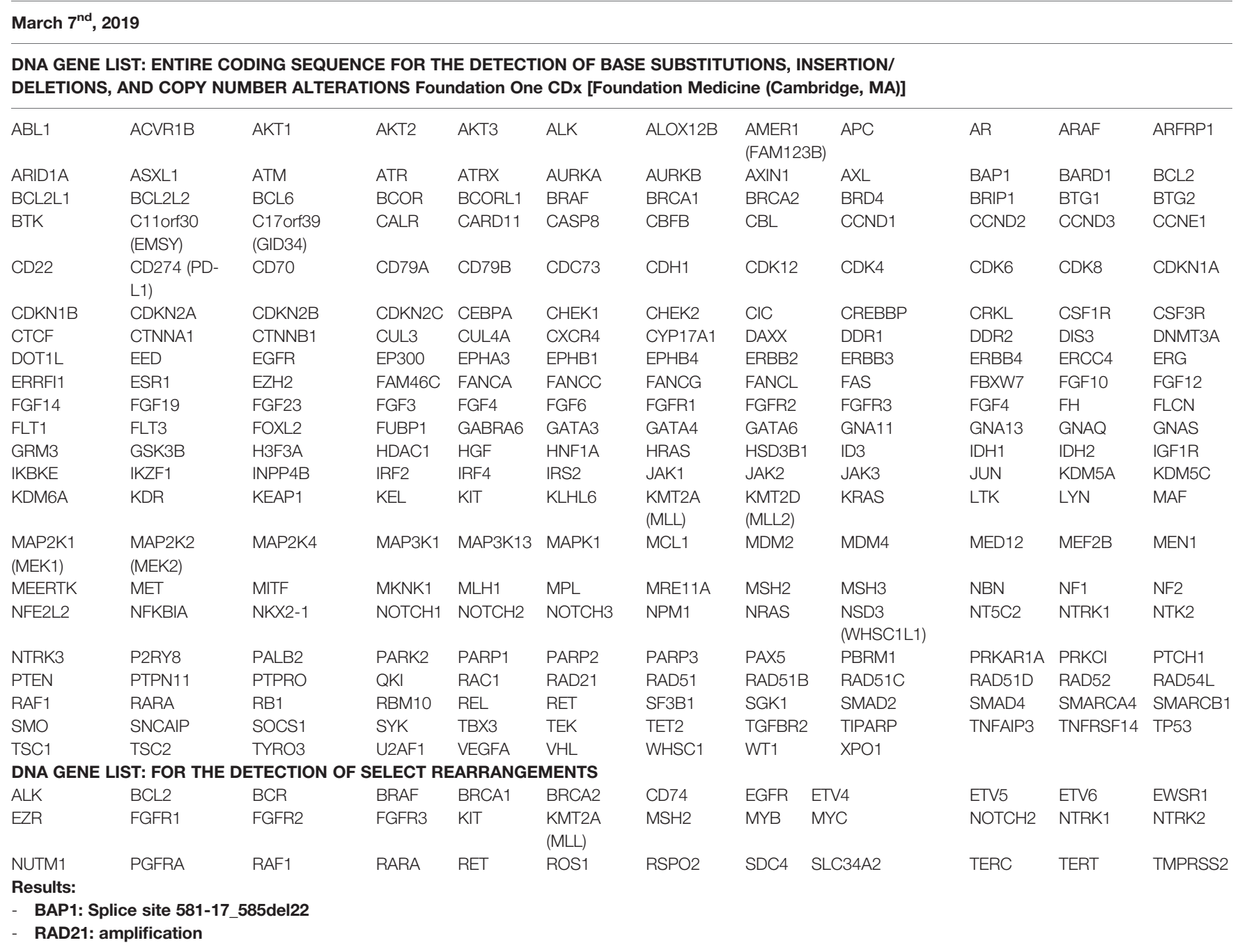

carcinogenesis in mesothelioma, lung adenocarcinoma and melanoma $(30,32,34,41)$. BAP1 mutations occur in $10-32 \%$ of ICC cases $(10,14,30,42-49)$. As a tumor suppressor gene, BAP1 seems to follow a classic two-hit model (Knudson model) in which probably the first hit involves loss of heterozygosity (LOH) induced by $3 \mathrm{p} 21$ deletion. The latter occurs in almost $50-$ $75 \%$ of ICCs (36). A subsequent mutation occurring in the remaining allele might lead to impairment of protein function and/or homeostasis (36). Protein function impairment by c.58117_585del22 is most likely to reflect a deletion in the 3'-splice site of BAP1. Previously a c.581(-5)_c.590delACTAGGGCCCTGGGG mutation has been reported causing a premature truncation of BAP1 (50). This type of alterations that disrupt the nuclear localizations signal (aminoacids 717-722) of BAP1 are predicted to be inactivating $(14,51)$.

As BAP1 interacts with BRCA1, several lines of evidence indicate that alterations in the BRCA-mediated DNA repair pathway confers sensitivity to PARP inhibitor administration (52). PARP inhibitors act through synthetic lethality, whereby genetic DNA repair defects are enhanced by drug-induced defects in a compensatory pathway (53). Carriers of heterozygous BRCA1/2 mutations are sensitive to PARP inhibitor treatment as they lose the wild-type allele during tumorigenesis and thereby become deficient of the homologous recombination (HR) pathway of double-strand break DNA repair by BRCA1/2-null status. Four PARP inhibitors, olaparib, rucaparib, niraparib, and talazoparib, have been approved by the U.S. Food and Drug Administration (FDA) and by the European Medicines Agency (EMA). In 2014, olaparib was approved as maintenance therapy for platinum-sensitive advanced ovarian cancer with germline mutations in BRCA1/2. In 2016, rucaparib was approved for advanced ovarian cancer with both germline and somatic BRCA1/2 mutations. In 2017 and 2018, olaparib, rucaparib, and niraparib were approved for the maintenance treatment of recurrent, epithelial ovarian, fallopian tube, or primary peritoneal cancer irrespective of the BRCA status. Last, in 2018, olaparib and talazoparib were approved for HER2-negative locally advanced or metastatic breast cancer with germline BRCA1/2 mutations. Besides in ovarian and breast cancer, PARP inhibitor efficacy has also been 


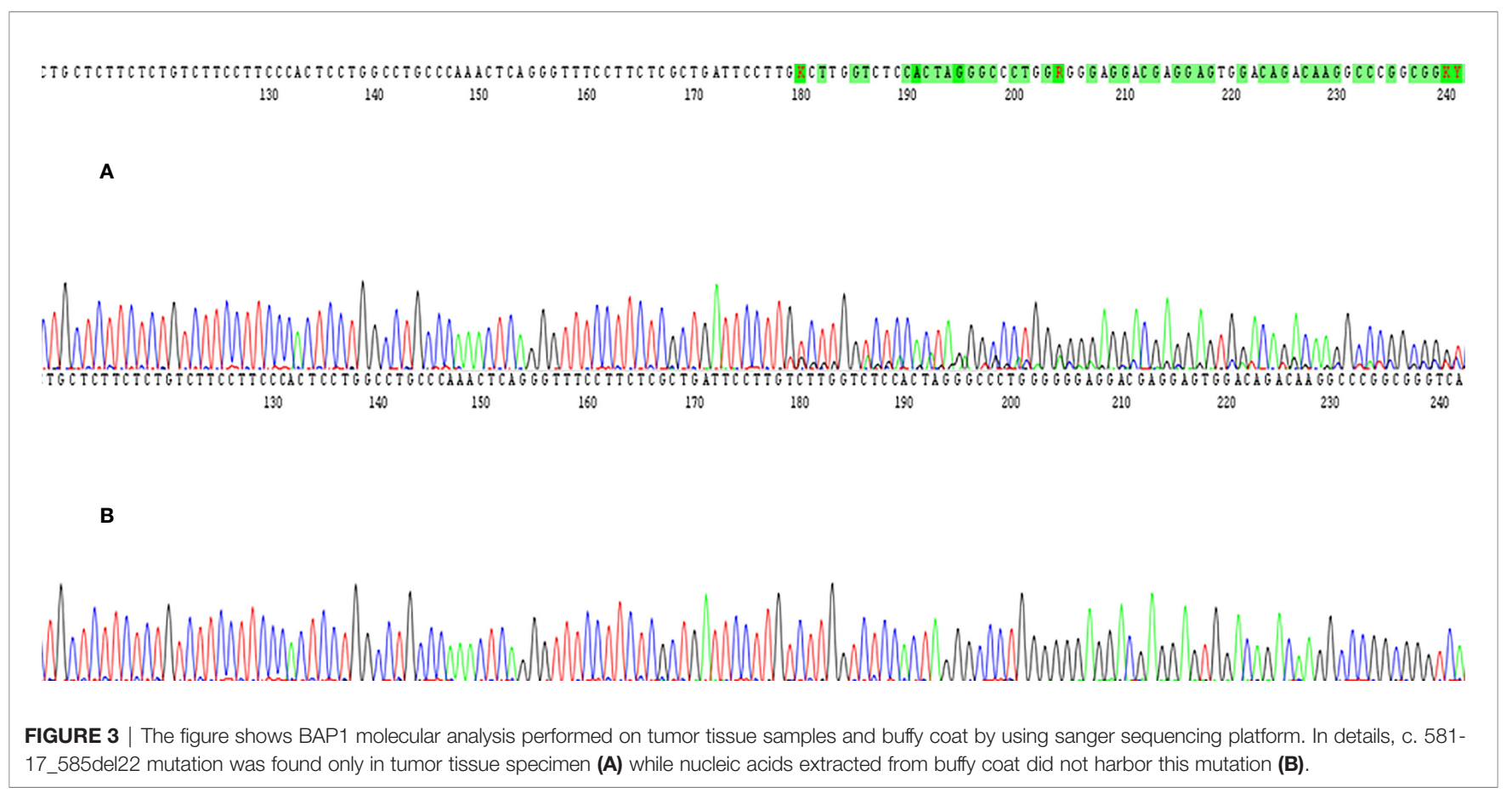

demonstrated in other types of cancer including prostate and pancreatic cancer, and small cell lung carcinoma, irrespective of the BRCA status (54-61). It has become clear that any form of HR deficiency in tumors that phenocopies BRCA1/2 mutations, often referred to as BRCAness, may sensitize cells to PARP inhibitors (62). Indeed mutations in DNA damage response genes such as ATM, PRKDC, ATR, RPA1, DSS1, NBN, RAD51, RAD54, CHEK1, CHEK2, FANC genes, ERCC1, POLB, FEN1, and $C D K 12$ have shown synthetic lethality in combination with PARP inhibitors (63-67).

BAP1 is a HR DNA repair component and its loss sensitizes cancer cells to DNA repair defects (28). Currently, further investigations are needed to establish the real efficacy of PARP inhibitor on BAP1 mutated cancer cells. Some studies on various types of BAP1 mutated cancer cell lines demonstrated the potential efficacy of PARP inhibitors (68-70). A synergistic effect of PARP inhibitor and gemcitabine is described in BAP1 deficient cholangiocarcinoma cell lines (71). As a result, PARP inhibitors are currently under investigation alone or in combination with other therapies in cancer patients harboring a BAP1 mutant tumor including ICC (ClinicalTrials.gov Identifier: NCT03207347, NCT03786796, NCT03531840, and NCT03375307).

In the current clinical case, we have shown that PARP inhibitor administration can be potentially effective in BAP1 mutated ICC. Chemotherapeutic agents, such as platinum compounds which induce double-strand DNA breaks, are usually utilized prior to PARP inhibition in order to enhance DNA damage and induce PARP inhibition-mediated cell death (72). In addition PARP inhibitors are currently administered after obtaining a disease control with platinum compounds (73, 74). In the present clinical case, the PARP inhibitor olaparib was effective in controlling tumor progression, even though the patient did not benefit from FOLFIRI administration, a combination of 5-FU and topoisomerase I inhibitor irinotecan. Irinotecan exerts its anticancer effects through induction of single- and double-strand DNA breaks. 5-FU is an antimetabolite drug that exerts its anticancer effects through inhibition of DNA synthesis by inhibition of thymidylate synthase and incorporation of its metabolites into RNA and DNA. One could speculate that efficacy to PARP inhibitor was not enhanced by FOLFIRI administration, but rather by the previous administration of cisplatin. Additional studies are needed to define the timing and schedule of DNA damaging agents for PARP inhibitor enhancement in BAP1 deficient tumors.

In addition to BAP1 mutations, many other molecular alterations have been described in ICC such as KRAS, BRAF, IDH1, IDH2, EGFR, FGFR2, ROS1, ARID1A, PBRM1, and $B R C A 1$ (8-16). These types of alterations are frequently mutually exclusive (8-16). In the current clinical case, BAP1 mutation is not associated with KRAS, BRAF, IDH1, IDH2, EGFR, FGFR2, ROS1, ARID1A, PBRM1, and BRCA1 alterations but with a RAD21 amplification. Further studies are needed to validate this type of association. RAD21 is a gene involved in the repair of DNA double-strand breaks, as well as in chromatid cohesion during mitosis $(75,76)$. Amplification of RAD21 is described in approximately $1.23 \%$ of cases reported in the AACR Project Genomics Evidence Neoplasia Information Exchange (AACR Project GENIE), including invasive breast carcinoma, prostate adenocarcinoma, lung adenocarcinoma and colon adenocarcinoma having the greatest prevalence (77). However, no prior data exists regarding RAD21 amplification in ICC. Whether RAD21 amplification might enhance the activity of a PARP inhibitor in BAP1 mutant ICC should be further investigated. 
Both BAP1 and RAD21 alterations were detected by utilizing NGS analysis. Patient's tumor tissue underwent analysis by several precision oncology testing methods to identify potentially oncogenic alterations. However, most of the tests performed did not detect any alterations. By comparing the results from the two most extensive tumor genomic profiles BAP1 was analyzed in both: the Foudation One CDx and Oncofocus test. However only the Foudation One CDx test was able to detect BAP1 and RAD21 alterations. These findings are likely to reflect the different methods utilized to detect potentially oncogenic alterations, the regions of the genes included in the analysis, the potential tumor heterogeneity especially with a low allele frequency of the variants and the percentage of tumor cells in the sample tested. Since there is no targeted regions for BAP1 it is unlikely that different NGS platforms only test selected exons. In our case the novel mutation c.58117_585del22 of BAP1 was localized on exon 8 of BAP1, at the boundary of intron 7. Most of the NGS platforms include 20-25bp in the vicinity of exons. However the Oncofocus ${ }^{\circledR}$ Test did not detect the c.581-17_585del22 alteration of BAP1 alteration most likely because this region of the gene was not included in the analysis. In contrast, the Foundation One CDx platform included in the analysis the full exonic region of $B A P 1$ besides including also $R A D 21$ in the analysis. Foundation One CDx report contains information only about the genomic findings without allele frequency values. As limit of detection range at nonhomopolymer context (insertion up to $42 \mathrm{bp}$ and deletion up to $276 \mathrm{bp}$ ) is $6-10 \%$, we can assume that the BAP1 c.581-17_585del mutated allele was present with a higher variant fraction in the metastatic tumor tissue analyzed. In addition, direct sequencing has a reported limit of detection of approximately $20 \%$ mutant alleles. In our case BAP1 sanger sequencing on primary ICC tumor tissue showed the unbalanced presence of the mutated allele, even if it is not possible to have a quantitative value, as with NGS or digital PCR, we can hypothesize an allele frequency close to the limit of detection. Therefore, we can assume that BAP1 c.581-17_585del mutated allele occurred with a high allele frequency, early in ICC oncogenesis.

In conclusion, genomic characterization of ICC tumors by NGS analysis can identify potential targetable oncogenic alterations in ICC, providing the possibility to improve patient survival. Specifically, BAP1 deletion and RAD21 amplification were identified and effectively targeted by PARP inhibitor administration. These results warrant further studies to define the role of PARP inhibitor in ICC harboring BAP1 and RAD21 alterations.

\section{REFERENCES}

1. Massarweh NN, El-Serag HB. Epidemiology of Hepatocellular Carcinoma and Intrahepatic Cholangiocarcinoma. Cancer Control (2017) 24:1-11. doi: 10.1177/1073274817729245 1073274817729245.

2. Bridgewater J, Galle PR, Khan SA, Llovet JM, Park J-W, Patel T, et al. Guidelines for the diagnosis and management of intrahepatic cholangiocarcinoma. J Hepatol (2014) 60:1268-89. doi: 10.1016/j.jhep. 2014.01.021

3. Rahnemai-Azar AA, Weisbrod A, Dillhoff M, Schmidt C, Pawlik TM. Intrahepatic cholangiocarcinoma: Molecular markers for diagnosis and prognosis. Surg Oncol (2017) 26:125-37. doi: 10.1016/j.suronc.2016.12.009

\section{DATA AVAILABILITY STATEMENT}

The original contributions presented in the study are included in the article/Supplementary Material. Further inquiries can be directed to the corresponding author.

\section{ETHICS STATEMENT}

Written informed consent was obtained from the patient for publication of this case report and any accompanying images.

\section{AUTHOR CONTRIBUTIONS}

Conception and design: FSa, SP, and UM. Acquisition of data: LL, VT, and FSa. Analysis and interpretation of data: FSa, AF, VC, FSc, and UM. Writing, review, and/or revision of the manuscript: FSa, LL, and CF. Administrative, technical, or material support (i.e., reporting or organizing data, constructing databases): LL, and VT. Study supervision: SP. Other (contributed clinical and pathological material; discussed results and implications of findings): SP, GT, and CF. All authors contributed to the article and approved the submitted version.

\section{FUNDING}

The work was supported by Ministero dell'Università e della Ricerca (Progetti di Rilevante Interesse Nazionale (PRIN), 2017, CODICE 2017PHRC8X_003) (to SP).

\section{ACKNOWLEDGEMENTS}

The authors wish to gratefully acknowledge the patient for allowing us to publish his clinical case.

\section{SUPPLEMENTARY MATERIAL}

The Supplementary Material for this article can be found online at: https://www.frontiersin.org/articles/10.3389/fonc. 2020. 567289/full\#supplementary-material

4. Valle J, Wasan H, Palmer DH, Cunningham D, Anthoney A, Maraveyas A, et al. Cisplatin plus gemcitabine versus gemcitabine for biliary tract cancer. N Engl J Med (2010) 362:1273-81. doi: 10.1056/NEJMoa0908721

5. Fujimura T, Fujisawa Y, Kambayashi Y, Aiba S. Significance of BRAF Kinase Inhibitors for Melanoma Treatment: From Bench to Bedside. Cancers (Basel) (2019) 11:1342. doi: 10.3390/cancers11091342

6. Schwartzberg L, Kim ES, Liu D, Schrag D. Precision Oncology: Who, How, What, When, and When Not? Am Soc Clin Oncol Educ Book (2017) 37:160-9. doi: 10.1200/EDBK_174176

7. Valle JW, Lamarca A, Goyal L, Barriuso J, Zhu AX. New Horizons for Precision Medicine in Biliary Tract Cancers. Cancer Discov (2017) 7:943-62. doi: 10.1158/2159-8290.CD-17-0245 
8. Arai Y, Totoki Y, Hosoda F, Shirota T, Hama N, Nakamura H, et al. Fibroblast growth factor receptor 2 tyrosine kinase fusions define a unique molecular subtype of cholangiocarcinoma. Hepatology (2014) 59:1427-34. doi: 10.1002/ hep. 26890

9. Gu T-L, Deng X, Huang F, Tucker M, Crosby K, Rimkunas V, et al. Survey of tyrosine kinase signaling reveals ROS kinase fusions in human cholangiocarcinoma. Plos One (2011) 6:e15640. doi: 10.1371/ journal.pone.0015640

10. Jiao Y, Pawlik TM, Anders RA, Selaru FM, Streppel MM, Lucas DJ, et al. Exome sequencing identifies frequent inactivating mutations in BAP1, ARID1A and PBRM1 in intrahepatic cholangiocarcinomas. Nat Genet (2013) 45:1470-3. doi: 10.1038/ng.2813

11. Moeini A, Sia D, Bardeesy N, Mazzaferro V, Llovet JM. Molecular Pathogenesis and Targeted Therapies for Intrahepatic Cholangiocarcinoma. Clin Cancer Res (2016) 22:291-300. doi: 10.1158/1078-0432.CCR-14-3296

12. Saha SK, Parachoniak CA, Ghanta KS, Fitamant J, Ross KN, Najem MS, et al. Mutant IDH inhibits HNF- $4 \alpha$ to block hepatocyte differentiation and promote biliary cancer. Nature (2014) 513:110-4. doi: 10.1038/nature13441

13. Yoshikawa D, Ojima H, Iwasaki M, Hiraoka N, Kosuge T, Kasai S, et al. Clinicopathological and prognostic significance of EGFR, VEGF, and HER2 expression in cholangiocarcinoma. Br J Cancer (2008) 98:418-25. doi: $10.1038 /$ sj.bjc. 6604129

14. Chan-On W, Nairismägi M-L, Ong CK, Lim WK, Dima S, Pairojkul C, et al. Exome sequencing identifies distinct mutational patterns in liver fluke-related and non-infection-related bile duct cancers. Nat Genet (2013) 45:1474-8. doi: $10.1038 /$ ng.2806

15. Andrici J, Goeppert B, Sioson L, Clarkson A, Renner M, Stenzinger A, et al. Loss of BAP1 Expression Occurs Frequently in Intrahepatic Cholangiocarcinoma. Med (Baltimore) (2016) 95:e2491. doi: 10.1097/ MD.0000000000002491

16. Sia D, Hoshida Y, Villanueva A, Roayaie S, Ferrer J, Tabak B, et al. Integrative molecular analysis of intrahepatic cholangiocarcinoma reveals 2 classes that have different outcomes. Gastroenterology (2013) 144:829-40. doi: 10.1053/ j.gastro.2013.01.001

17. Javle M, Lowery M, Shroff RT, Weiss KH, Springfeld C, Borad MJ, et al. Phase II Study of BGJ398 in Patients With FGFR-Altered Advanced Cholangiocarcinoma. J Clin Oncol (2018) 36:276-82. doi: 10.1200/ JCO.2017.75.5009

18. Nakanishi Y, Akiyama N, Tsukaguchi T, Fujii T, Sakata K, Sase H, et al. The fibroblast growth factor receptor genetic status as a potential predictor of the sensitivity to CH5183284/Debio 1347, a novel selective FGFR inhibitor. Mol Cancer Ther (2014) 13:2547-58. doi: 10.1158/1535-7163.MCT-14-0248

19. Goyal L, Shi L, Liu LY, Fece de la Cruz F, Lennerz JK, Raghavan S, et al. TAS120 Overcomes Resistance to ATP-Competitive FGFR Inhibitors in Patients with FGFR2 Fusion-Positive Intrahepatic Cholangiocarcinoma. Cancer Discov (2019) 9:1064-79. doi: 10.1158/2159-8290.CD-19-0182

20. Jensen DE, Rauscher FJ. BAP1, a candidate tumor suppressor protein that interacts with BRCA1. Ann N Y Acad Sci (1999) 886:191-4. doi: 10.1111/ j.1749-6632.1999.tb09414.x

21. Jensen DE, Proctor M, Marquis ST, Gardner HP, Ha SI, Chodosh LA, et al. BAP1: a novel ubiquitin hydrolase which binds to the BRCA1 RING finger and enhances BRCA1-mediated cell growth suppression. Oncogene (1998) 16:1097-112. doi: 10.1038/sj.onc.1201861

22. Baughman JM, Rose CM, Kolumam G, Webster JD, Wilkerson EM, Merrill AE, et al. NeuCode Proteomics Reveals Bap1 Regulation of Metabolism. Cell Rep (2016) 16:583-95. doi: 10.1016/j.celrep.2016.05.096

23. Bononi A, Giorgi C, Patergnani S, Larson D, Verbruggen K, Tanji M, et al. BAP1 regulates IP3R3-mediated Ca2+ flux to mitochondria suppressing cell transformation. Nature (2017) 546:549-53. doi: 10.1038/nature22798

24. Eletr ZM, Wilkinson KD. An emerging model for BAP1's role in regulating cell cycle progression. Cell Biochem Biophys (2011) 60:3-11. doi: 10.1007/ s12013-011-9184-6

25. Machida YJ, Machida Y, Vashisht AA, Wohlschlegel JA, Dutta A. The deubiquitinating enzyme BAP1 regulates cell growth via interaction with HCF-1. J Biol Chem (2009) 284:34179-88. doi: 10.1074/jbc.M109.046755

26. Ruan H-B, Han X, Li M-D, Singh JP, Qian K, Azarhoush S, et al. O-GlcNAc transferase/host cell factor $\mathrm{C} 1$ complex regulates gluconeogenesis by modulating PGC-1 $\alpha$ stability. Cell Metab (2012) 16:226-37. doi: 10.1016/ j.cmet.2012.07.006

27. Xu J, Kadariya Y, Cheung M, Pei J, Talarchek J, Sementino E, et al. Germline Mutation of Bap1 Accelerates Development of Asbestos-Induced Malignant Mesothelioma. Cancer Res (2014) 74:4388-97. doi: 10.1158/0008-5472.CAN14-1328

28. Yu H, Pak H, Hammond-Martel I, Ghram M, Rodrigue A, Daou S, et al. Tumor suppressor and deubiquitinase BAP1 promotes DNA double-strand break repair. Proc Natl Acad Sci USA (2014) 111:285-90. doi: 10.1073/ pnas. 1309085110

29. Yoshida K, Miki Y. Role of BRCA1 and BRCA2 as regulators of DNA repair, transcription, and cell cycle in response to DNA damage. Cancer Sci (2004) 95:866-71. doi: 10.1111/j.1349-7006.2004.tb02195.x

30. Di Nunno V, Frega G, Santoni M, Gatto L, Fiorentino M, Montironi R, et al. BAP1 in solid tumors. Future Oncol (2019) 15:2151-62. doi: 10.2217/fon2018-0915

31. Carbone M, Yang H, Pass HI, Krausz T, Testa JR, Gaudino G. BAP1 and cancer. Nat Rev Cancer (2013) 13:153-9. doi: 10.1038/nrc3459

32. Cheung M, Talarchek J, Schindeler K, Saraiva E, Penney LS, Ludman M, et al. Further evidence for germline BAP1 mutations predisposing to melanoma and malignant mesothelioma. Cancer Genet (2013) 206:206-10. doi: 10.1016/ j.cancergen.2013.05.018

33. Pilarski R, Rai K, Cebulla C, Abdel-Rahman M. BAP1 Tumor Predisposition Syndrome, in: GeneReviews ${ }^{\circledR}$. Seattle (WA: University of Washington, Seattle. Available at: http://www.ncbi.nlm.nih.gov/books/NBK390611/ (Accessed July $28,2020)$

34. Abdel-Rahman MH, Pilarski R, Cebulla CM, Massengill JB, Christopher BN, Boru G, et al. Germline BAP1 mutation predisposes to uveal melanoma, lung adenocarcinoma, meningioma, and other cancers. J Med Genet (2011) 48:8569. doi: 10.1136/jmedgenet-2011-100156

35. Carbone M, Flores EG, Emi M, Johnson TA, Tsunoda T, Behner D, et al. Combined Genetic and Genealogic Studies Uncover a Large BAP1 Cancer Syndrome Kindred Tracing Back Nine Generations to a Common Ancestor from the 1700s. PloS Genet (2015) 11:e1005633. doi: 10.1371/ journal.pgen.1005633

36. Murali R, Wiesner T, Scolyer RA. Tumours associated with BAP1 mutations. Pathology (2013) 45:116-26. doi: 10.1097/PAT.0b013e32835d0efb

37. Kobrinski DA, Yang H, Kittaneh M. BAP1: role in carcinogenesis and clinical implications. Transl Lung Cancer Res (2020) 9:S60-6. doi: 10.21037/ tlcr.2019.11.24

38. Carbone M, Ferris LK, Baumann F, Napolitano A, Lum CA, Flores EG, et al. BAP1 cancer syndrome: malignant mesothelioma, uveal and cutaneous melanoma, and MBAITs. J Transl Med (2012) 10:179. doi: 10.1186/14795876-10-179

39. Ewens KG, Kanetsky PA, Richards-Yutz J, Purrazzella J, Shields CL, Ganguly T, et al. Chromosome 3 status combined with BAP1 and EIF1AX mutation profiles are associated with metastasis in uveal melanoma. Invest Ophthalmol Vis Sci (2014) 55:5160-7. doi: 10.1167/iovs.14-14550

40. Ewens KG, Lalonde E, Richards-Yutz J, Shields CL, Ganguly A. Comparison of Germline versus Somatic BAP1 Mutations for Risk of Metastasis in Uveal Melanoma. BMC Cancer (2018) 18:1172. doi: 10.1186/s12885-018-5079-x

41. Masoomian B, Shields JA, Shields CL. Overview of BAP1 cancer predisposition syndrome and the relationship to uveal melanoma. J Curr Ophthalmol (2018) 30:102-9. doi: 10.1016/j.joco.2018.02.005

42. Bogenberger JM, DeLeon TT, Arora M, Ahn DH, Borad MJ. Emerging role of precision medicine in biliary tract cancers. NPJ Precis Oncol (2018) 2:21. doi: 10.1038/s41698-018-0064-z

43. Simbolo M, Fassan M, Ruzzenente A, Mafficini A, Wood LD, Corbo V, et al. Multigene mutational profiling of cholangiocarcinomas identifies actionable molecular subgroups. Oncotarget (2014) 5:2839-52. doi: 10.18632/ oncotarget.1943

44. Fujimoto A, Furuta M, Shiraishi Y, Gotoh K, Kawakami Y, Arihiro K, et al. Whole-genome mutational landscape of liver cancers displaying biliary phenotype reveals hepatitis impact and molecular diversity. Nat Commun (2015) 6:6120. doi: 10.1038/ncomms7120

45. Ross JS, Wang K, Gay L, Al-Rohil R, Rand JV, Jones DM, et al. New routes to targeted therapy of intrahepatic cholangiocarcinomas revealed by next- 
generation sequencing. Oncologist (2014) 19:235-42. doi: 10.1634/ theoncologist.2013-0352

46. Churi CR, Shroff R, Wang Y, Rashid A, Kang HC, Weatherly J, et al. Mutation Profiling in Cholangiocarcinoma: Prognostic and Therapeutic Implications. PloS One (2014) 9:e115383. doi: 10.1371/journal.pone.0115383

47. Zou S, Li J, Zhou H, Frech C, Jiang X, Chu JSC, et al. Mutational landscape of intrahepatic cholangiocarcinoma. Nat Commun (2014) 5:5696. doi: 10.1038/ ncomms6696

48. Sia D, Losic B, Moeini A, Cabellos L, Hao K, Revill K, et al. Massive parallel sequencing uncovers actionable FGFR2 - PPHLN1 fusion and ARAF mutations in intrahepatic cholangiocarcinoma. Nat Commun (2015) 6:6087. doi: $10.1038 /$ ncomms 7087

49. Borad MJ, Champion MD, Egan JB, Liang WS, Fonseca R, Bryce AH, et al. Integrated genomic characterization reveals novel, therapeutically relevant drug targets in FGFR and EGFR pathways in sporadic intrahepatic cholangiocarcinoma. PloS Genet (2014) 10:e1004135. doi: 10.1371/ journal.pgen. 1004135

50. Farshidfar F, Zheng S, Gingras M-C, Newton Y, Shih J, Robertson AG, et al. Integrative Genomic Analysis of Cholangiocarcinoma Identifies Distinct IDH-Mutant Molecular Profiles. Cell Rep (2017) 18:2780-94. doi: 10.1016/ j.celrep.2017.02.033

51. Ventii KH, Devi NS, Friedrich KL, Chernova TA, Tighiouart M, Van Meir EG, et al. BRCA1-associated protein-1 is a tumor suppressor that requires deubiquitinating activity and nuclear localization. Cancer Res (2008) 68:6953-62. doi: 10.1158/0008-5472.CAN-08-0365

52. Kamel D, Gray C, Walia JS, Kumar V. PARP Inhibitor Drugs in the Treatment of Breast, Ovarian, Prostate and Pancreatic Cancers: An Update of Clinical Trials. Curr Drug Targets (2018) 19:21-37. doi: 10.2174/13894501186661 70711151518

53. Lord CJ, Ashworth A. PARP inhibitors: Synthetic lethality in the clinic. Science (2017) 355:1152-8. doi: 10.1126/science.aam7344

54. Weaver AN, Yang ES. Beyond DNA Repair: Additional Functions of PARP-1 in Cancer. Front Oncol (2013) 3:290. doi: 10.3389/fonc.2013.00290

55. Sonnenblick A, de Azambuja E, Azim HA, Piccart M. An update on PARP inhibitors-moving to the adjuvant setting. Nat Rev Clin Oncol (2015) 12:2741. doi: $10.1038 /$ nrclinonc. 2014.163

56. Mirza MR, Pignata S, Ledermann JA. Latest clinical evidence and further development of PARP inhibitors in ovarian cancer. Ann Oncol (2018) 29:1366-76. doi: 10.1093/annonc/mdy174

57. Franzese E, Centonze S, Diana A, Carlino F, Guerrera LP, Napoli MD, et al. PARP inhibitors in ovarian cancer. Cancer Treat Rev (2019) 73:1-9. doi: 10.1016/j.ctrv.2018.12.002

58. Keung MYT, Wu Y, Vadgama JV. PARP Inhibitors as a Therapeutic Agent for Homologous Recombination Deficiency in Breast Cancers. J Clin Med (2019) 8:435. doi: $10.3390 / \mathrm{jcm} 8040435$

59. Mateo J, Lord CJ, Serra V, Tutt A, Balmaña J, Castroviejo-Bermejo M, et al. de Bono JS. A decade of clinical development of PARP inhibitors in perspective. Ann Oncol (2019) 30:1437-47. doi: 10.1093/annonc/mdz192

60. Pant S, Maitra A, Yap TA. PARP inhibition - opportunities in pancreatic cancer. Nat Rev Clin Oncol (2019) 16:595-6. doi: 10.1038/s41571-019-0257-6

61. Pilié PG, Gay CM, Byers LA, O'Connor MJ, Yap TA. PARP Inhibitors: Extending Benefit Beyond BRCA-Mutant Cancers. Clin Cancer Res (2019) 25:3759-71. doi: 10.1158/1078-0432.CCR-18-0968

62. Lord CJ, Ashworth A. BRCAness revisited. Nat Rev Cancer (2016) 16:110-20. doi: $10.1038 / \mathrm{nrc} .2015 .21$

63. Bryant HE, Helleday T. Inhibition of poly (ADP-ribose) polymerase activates ATM which is required for subsequent homologous recombination repair. Nucleic Acids Res (2006) 34:1685-91. doi: 10.1093/nar/gkl108

64. McCabe N, Turner NC, Lord CJ, Kluzek K, Białkowska A, Swift S, et al. Deficiency in the Repair of DNA Damage by Homologous Recombination and Sensitivity to Poly(ADP-Ribose) Polymerase Inhibition. Cancer Res (2006) 66:8109-15. doi: 10.1158/0008-5472.CAN-06-0140
65. Murai J, Huang SN, Das BB, Renaud A, Zhang Y, Doroshow JH, et al. Trapping of PARP1 and PARP2 by Clinical PARP Inhibitors. Cancer Res (2012) 72:5588-99. doi: 10.1158/0008-5472.CAN-12-2753

66. Postel-Vinay S, Bajrami I, Friboulet L, Elliott R, Fontebasso Y, Dorvault N, et al. A high-throughput screen identifies PARP1/2 inhibitors as a potential therapy for ERCC1-deficient non-small cell lung cancer. Oncogene (2013) 32:5377-87. doi: 10.1038/onc.2013.311

67. Bajrami I, Frankum JR, Konde A, Miller RE, Rehman FL, Brough R, et al. Genome-wide Profiling of Genetic Synthetic Lethality Identifies CDK12 as a Novel Determinant of PARP1/2 Inhibitor Sensitivity. Cancer Res (2014) 74:287-97. doi: 10.1158/0008-5472.CAN-13-2541

68. Peña-Llopis S, Vega-Rubín-de-Celis S, Liao A, Leng N, Pavía-Jiménez A, Wang S, et al. BAP1 loss defines a new class of renal cell carcinoma. Nat Genet (2012) 44:751-9. doi: 10.1038/ng.2323

69. Parrotta R, Okonska A, Ronner M, Weder W, Stahel R, Penengo L. FelleyBosco E. A Novel BRCA1-Associated Protein-1 Isoform Affects Response of Mesothelioma Cells to Drugs Impairing BRCA1-Mediated DNA Repair. J Thorac Oncol (2017) 12:1309-19. doi: 10.1016/j.jtho.2017.03.023

70. Rathkey D, Khanal M, Murai J, Zhang J, Sengupta M, Jiang Q, et al. Sensitivity of Mesothelioma Cells to PARP Inhibitors Is Not Dependent on BAP1 but Is Enhanced by Temozolomide in Cells With High-Schlafen 11 and Low-O6-methylguanine-DNA Methyltransferase Expression. J Thorac Oncol (2020) 15:843-59. doi: 10.1016/j.jtho.2020.01.012

71. Parasramka M, Yan IK, Wang X, Nguyen P, Matsuda A, Maji S, et al. BAP1 dependent expression of long non-coding RNA NEAT-1 contributes to sensitivity to gemcitabine in cholangiocarcinoma. Mol Cancer (2017) 16:22. doi: 10.1186/s12943-017-0587-x

72. Mylavarapu S, Das A, Roy M. Role of BRCA Mutations in the Modulation of Response to Platinum Therapy. Front Oncol (2018) 8:16. doi: 10.3389/ fonc.2018.00016

73. Pennington KP, Walsh T, Harrell MI, Lee MK, Pennil CC, Rendi MH, et al. Germline and somatic mutations in homologous recombination genes predict platinum response and survival in ovarian, fallopian tube, and peritoneal carcinomas. Clin Cancer Res (2014) 20:764-75. doi: 10.1158/1078-0432.CCR13-2287

74. Wang ZC, Birkbak NJ, Culhane AC, Drapkin R, Fatima A, Tian R, et al. Profiles of Genomic Instability in High-Grade Serous Ovarian Cancer Predict Treatment Outcome. Clin Cancer Res (2012) 18:5806-15. doi: 10.1158/10780432.CCR-12-0857

75. Peters J-M, Nishiyama T. Sister Chromatid Cohesion. Cold Spring Harb Perspect Biol (2012) 4:a011130. doi: 10.1101/cshperspect.a011130

76. Hill VK, Kim J-S, Waldman T. Cohesin Mutations in Human Cancer. Biochim Biophys Acta (2016) 1866:1-11. doi: 10.1016/j.bbcan.2016.05.002

77. AACR Project GENIE Consortium. AACR Project GENIE: Powering Precision Medicine through an International Consortium. Cancer Discov (2017) 7:818-31. doi: 10.1158/2159-8290.CD-17-0151

Conflict of Interest: UM reports personal fees (as speaker bureau or advisor) from Boehringer Ingelheim, AstraZeneca, Roche, MSD, Amgen and Merck, unrelated to the current work.

The remaining authors declare that the research was conducted in the absence of any commercial or financial relationships that could be construed as a potential conflict of interest.

Copyright (c) 2020 Sabbatino, Liguori, Malapelle, Schiavi, Tortora, Conti, Filippelli, Tortora, Ferrone and Pepe. This is an open-access article distributed under the terms of the Creative Commons Attribution License (CC BY). The use, distribution or reproduction in other forums is permitted, provided the original author(s) and the copyright owner(s) are credited and that the original publication in this journal is cited, in accordance with accepted academic practice. No use, distribution or reproduction is permitted which does not comply with these terms. 